Wissenschaft und Fortbildung

\title{
Oxalsäurebestimmung im Plasma mit Oxalat-Oxidase. Untersuchungen bei Gesunden und bei Patienten mit terminaler Niereninsuffizienz vor und nach Dialyse
}

\author{
U. Rehmert, K. Wicher, W. Ruge und J. Bahimann \\ Zentrum Innere Medizin und Dermatologie, Medizinische Hochschule Hannover (Geschäftsführender Direktor Prof. Dr. F. Hartmann)
}

\section{Zusammenfassung:}

Es wird eine enzymatische Bestimmung der Oxalsäurekonzentration im Plasma mittels Oxalat-Oxidase (E.C. 1.2.3.4.), basierend auf der Methode von Sugiura et al. (1), beschrieben. Sie zeichnet sich durch eine hohe Spezifität, große Empfindlichkeit und Genauigkeit sowie durch einfache und schnelle Handhabung aus. Die mögliche Neubildung von Oxalsäure aus Glyoxylsäure nach der Blutentnahme wird durch den Zusatz von Inhibitoren verhindert. Die Oxalsäurekonzentration im Plasma liegt bei 15 stoffwechselgesunden Probanden im Bereich von 0,08 bis $0.25 \mathrm{mg} / \mathrm{dl}$ (7.0-22,0 $\mu \mathrm{mol} / \mathrm{l})$ und bei 25 Patienten mit terminaler Niereninsuffizienz zwischen 0,39 und 1,36 mg/dl (34,5$120,4 \mu \mathrm{mol} / 1)$. Nach Dialyse ist die Oxalsäurekonzentration auf den Medianwert von $\tilde{x}=56,3 \%$ der Konzentration vor Dialyse gesunken. Die endogene Kreatinin-Clearance der niereninsuffizienten Patienten zeigt einen stärkeren Abfall als die Oxalsäure-Clearance.

\section{Schlüsse/wörter:}

Oxalat-Oxidase - Oxalose - Oxalsäure - Oxalsäure-Clearance - terminale Niereninsuffizienz

\begin{abstract}
Summary:
A method for the determination of human plasma oxalate concentration with oxalate oxidase (E.C. 1.2.3.4.), based on the method by Sugiura (1). is described. It shows a high specificity, sensitivity, exactness and a quick and simple handling. The possible conversion of glyoxalate to oxalate in whole blood is prevented by addition of inhibitors. The plasma oxalate concentration of 15 normal subjects is found to be $0.08-0.25 \mathrm{mg} / \mathrm{dl}(7.0-22.0 \mu \mathrm{mol} / \mathrm{l})$, that of 25 patients with chronic renal failure in the range from $0.39-1.36 \mathrm{mg} / \mathrm{dl}(34.5-120.4 \mu \mathrm{mol} / 1)$. After dialysing the oxalate concentration falls to $\bar{x}=56.3 \%$ of the concentration before dialysing. The creatinin clearance of those patients with chronic renal failure shows a higher decrease than the oxalate clearance.
\end{abstract}

\section{Keywords:}

Oxalate oxidase - oxalose - oxalic acid - oxalate clearance - chronic renal failure

\section{Einleitung}

Die Messung der Oxalsäurekonzentration im Blut ist für die Erkennung einer primären Hyperoxalurie im Stadium einer terminalen Niereninsuffizienz voǹ entscheidender Bedeutung. Aber auch bei Niereninsuffizienzen anderer Átiologie läßt sich aus der Höhe des Plasmaoxalatspiegels möglicherweise das Risiko für das Auftreten von Oxalatniederschlägén in Organen ablesen. Zusätzliche Informationen kann die Bestimmung bei den verschiedenen Formen der sekundären Hyperoxalurie und bei Oxalatsteinbildnern liefern. Während für die Messung der Oxalsäurekonzentration im Urin reproduzierbare, einfache Verfahren zur Verfügung stehen (2-4), bemüht man sich um eine allgemein durchführbare und spezifische Methode für die klinische Routinediagnostik zur Bestimmung der Oxalsäurekonzentration im Plasma. 


\section{Methodik}

\section{Probanden}

Es wird die Oxalsäure- und Kreatininkonzentration im Plasma und Urin bei 15 stoffwechselgesunden Probanden unterschiedlichen Alters ohne Diätrestriktion sowie bei 25 Patienten mit terminaler Niereninsuffizienz untersucht. Die Patienten sind 1 bis 8 Jahre in regelmäßiger Hämodialysebehandlung. Der Altersbereich liegt zwischen 24 und 70 Jahren, das Körpergewicht beträgt zwischen 53 und $80 \mathrm{~kg}$. Die Hämodia. lyse wird über $5 \mathrm{Std}$. mit einem mittleren Blutdurchfluß von $300 \mathrm{ml} / \mathrm{min}$ und einem Dialysatfluß von $600 \mathrm{ml} / \mathrm{min}$ unter Einsatz von Dialysatoren mit $1,3 \mathrm{~m}^{2}$ Cuprophanmembran durchgeführ. Bei 17 Patienten werden die Messungen vor Beginn und nach Beendigung der Dialyse, bei 8 Patienten nur vor der Dialyse vorgenommen. 10 der 25 Patienten weisen eine Resturinausscheidung auf, während die übrigen anurisch sind.

\section{Material}

2 - Hydrazono - 2,3 - dihydro - 3 - methylbenzothiazolhydrochlorid zur Analyse (MBTH), Merck Art. 4527; Peroxidase Typ II (POD), Sigma No. P 8250; N,N-Dimethylanilin zur Synthese (DMA), Merck-Schuchardt Art. 803060; OxalatOxidase aus Gerstenkeimlingen, Boehringer, Mannheim, Best.-Nr. 567698; Oxalsäure suprapur, Merck Art. 489; Borsäure zur Analyse; Allopurinol, Sigma No. A 8003; DL- $\beta$ Phenylmilchsäure, Sigma P 7251 ; Kreatinin UV-Test enzymatisch, Boehringer, Best.-Nr. 166413: Natr. citric neutr. (Erg. B. 6) ; $3,8 \mathrm{~g}$ in Aqua bidest. ad $100 \mathrm{ml}$, Braun-Melsungen, Best.Nr. 88407-A.

\section{Durchführung}

Die bei Sugiura (1) angegebenen Konzentrationen der Peroxidase und der Oxalat-Oxidase reichen nicht aus, um eine lineare Beziehung zwischen Oxalsäurekonzentration und Extinktion zu ergeben. Das Verfahren nach Sugiura wird in folgender Weise modifiziert:

- Versetzen des Probanden- bzw. Patientenblutes im Verhältnis 1:4 mit Natriumzitrat und anschließendes Zentrifugieren.

\section{Extinktion}

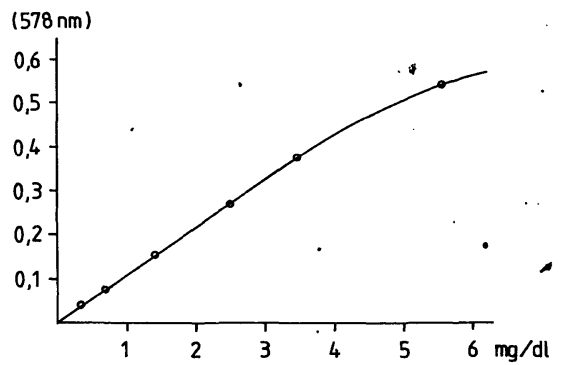

Abb. 1: Abhängigkeit der Extinktion von der OxalsäureKonzentration (mg/dl)

\section{- Doppelte Konzentrationen der Peroxidase und der Oxalat- Oxidase.}

- Bernsteinsäure-Puffer nach Gomori (5): $25 \mathrm{ml}$ einer $0,2 \mathrm{~mol} / \mathrm{/}$ Bernsteinsäure $(23,62 \mathrm{~g} / \mathrm{l})$ werden mit $0.2 \mathrm{~mol} / 1$ $\mathrm{NaOH}$ auf $\mathrm{pH}$-Wert 3,5 eingestellt und mit quarzdest. Wasser auf $100 \mathrm{ml}$ aufgefüllt.

- Zusatz einer Inhibitorlösung (71,9 mmol $=4,44 \mathrm{~g}$ Borsäure: $1.76 \mathrm{mmol}=240 \mathrm{mg}$ Allopurinol; $27,1 \mathrm{mmol}=4.5 \mathrm{~g} \mathrm{DL}$ $\beta$-Phenylmilchsäure in $100 \mathrm{ml}$ Wasser gelöst und auf $\mathrm{pH} 7,4$ eingestellt) zum Blut.

\section{- Messung der Extinktion bei $578 \mathrm{~nm}$.}

In einer Spritze werden $0,4 \mathrm{ml}$ Natriumzitrat - Lösung aufgezogen, mit Patientenblut auf $2 \mathrm{ml}$ aufgefülit und sofort mit $0,2 \mathrm{ml}$ Inhibitor-Lösung versetzt. Nach 3 minütigem Zentrifugieren bei $1000 \times \mathrm{g}$ wird das Plasma abgehoben und kühl gelagert $\left(4-8^{\circ} \mathrm{C}\right)$. Als Reagenzgemisch werden $125 \mu \mathrm{l}$ DMA, $5 \mathrm{mg}$ MBTH und $200 \mathrm{U}$ POD in $100 \mathrm{ml}$ des Puffers gelöst. $2,4 \mathrm{ml}$ des Reagenzgemisches werden $50 \mu$ l des Zitratplasmas zugefügt und für $3 \mathrm{~min}$ bei $37^{\circ} \mathrm{C}$ vorinkubiert. Danach werden $50 \mu \mathrm{l}$ der Oxalat-Oxidase- $\mathrm{Lsg}$. $(0,56 \mathrm{U} / \mathrm{ml})$ zúgesetzt und für weitere $30 \mathrm{~min}$ bei gleicher Temperatur inkubiert und anschließend im Eisbad gekühlt. Die Messung der Extinktion erfolgt mit einem Eppendorf-Photometer (Typ $1100 \mathrm{M}$ ) bei $578 \mathrm{~nm}$ und einer Schichtdicke von $2 \mathrm{~cm}$. Zur Bestimmung der Wiederfindung werden $7 \mu \mathrm{g}$ wasserfreie Oxalsäure pro $\mathrm{ml}$ Zitratplasma zugesetzt.

Die Bestimmung der Oxalsäurekonzentration im Urin erfolgt photometrisch mit Chromotropsäure nach einem Verfahren von Hodgkinson und Williams (4).

Die Kreatininkonzentrationen im Plasma sowie im Urin werden mit einem Kreatinin-UV-Test gemessen.

\section{Ergebnisse}

\section{Linearität}

Die Extinktion bei $578 \mathrm{~nm}$ verläuft linear zur OxalsäureKonzentration im Bereich von $0-3,5 \mathrm{mg} / \mathrm{dl}$. Ab $3,5 \mathrm{mg} / \mathrm{dl}$ nimmt die Extinktion im Verhältnis zur Konzentration leicht ab. Die klinisch relevanten Werte liegen im linearen Bereich der Funktionsverlaufskurve (Abb. 1).

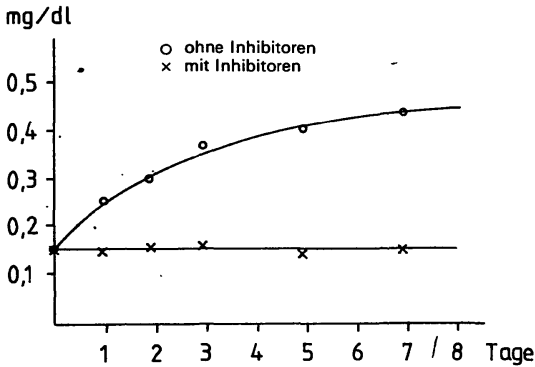

Abb. 2: Zeitliche Abhängigkeit der Oxalsäure-Konzentration mit und ohne Inhibitoren 


\section{Wiederfindung}

Die Wiederfindung dem Plasma zugesetzter Oxalsäure beträgt $98,9 \pm 6,95 \%\left(\bar{x} \pm S_{x} ; n=40\right)$.

\section{Reproduzierbarkeit der Messungen}

Zur Bestimmung der Reproduzierbarkeit der Messungen wird das Zitratplasma eines Patienten 10 mal gemessen. Der Variationskoeffizient in Serie beträgt $\mathrm{VK}_{\mathrm{s}}=\mathbf{2 , 7 7 \%}$.

\section{Einfluß von Inhibitoren}

Es wird die Oxalsäurekonzentration eines Zitratplasmas ohne Inhibitoren am 1., 2., 3., 5. und 7. Tag nach Blutentnahme gemessen (Abb. 2).

Die Oxalsäurekonzentration steigt im Plasma ohne Inhibitoren im Laufe der Zeit an, erreicht am 7. Tag etwa den dreifachen Ausgangswert, um sich dann anscheinend einem Höchstwert asymptotisch zu nähern. Dagegen bleibt die Konzentration im Plasma mit Inhibitoren im Rahmen der Meßgenauigkeit konstant.

\section{Normbereich}

Die Meßergebnisse von 15 stoffwechselgesunden Probanden ohne Diätrestriktionen zeigt Tab. 1.

\section{Niereninsuffizienz}

Die Werte der Oxalsäurekonzentration bei 25 Patienten mit terminaler Niereninsuffizienz sind in Tab. 2 dargestellt. Der Medianwert liegt 5,5mal so hoch wie derjenige des Normalkollektivs (Abb. 3).

Bei 17 dieser 25 Patienten wird der Plasmaoxalsäurespiegel vor und nach Dialyse gemessen (Tab. 2 und Abb. 4). Durch die Dialyse kommt es zu einem Abfall auf $56,3 \%$ des prädialytischen Wertes.

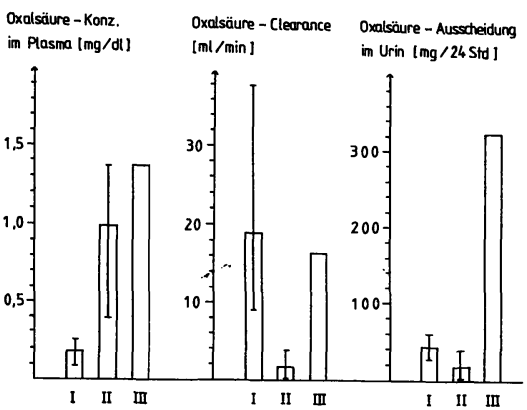

Abb. 3: Oxalsäure-Konzentration im Plasma, Oxalsäure-Clearance und Oxa/säure-Ausscheidung im Urin bei I stoffwechselgesunden Probanden ( $n=15)$, II Patienten mit terminaler Niereninsuffizienz $(n=25$ bei Plasmakonzentration, $n=10$ bei Clearance und Urin-Ausscheidung) und III einer Patientin mit Oxalat-Niederschlägen in Organen $(n=1)$. Dargestel/t sind Medianwert, Minimum und Maximum

Der Medianwert der Oxalsäure-Clearance beträgt bei den 10 Patienten mit Restharnausscheidung bei terminaler Niereninsuffizienz 1,68 ml/min (Tab. 3) und ist damit auf $8,9 \%$ des Wertes bei Gesunden abgefallen (Abb. 3). Der Medianwert der Kreatinin-Clearance beträgt bei diesen Patienten $0,91 \mathrm{ml} / \mathrm{min}$. Das Verhältnis der Oxalsäure-Clearance zur Kreatinin-Clearance liegt bei den niereninsuffizienten Patienten zwischen 0,42 und $2,61(\tilde{x}=1,42)$ (Tab. 3$)$, während es bei dem stoffwechselgesunden Kollektiv $0,09-0,33(\tilde{x}=$ $0,15)$ beträgt (Tab. 1).

Tab. 1: Meßergebnisse bei stoffwechselgesunden Probanden

\begin{tabular}{llccccc}
\hline & $\mathrm{n}$ & Minimum & 1s & Median & 3S & Maximum \\
\hline Oxalsäurekonzentration im Plasma [mg/dl] & 15 & 0,08 & 0,11 & 0,18 & 0,21 & 0,25 \\
Oxalsäureausscheidung im Urin [mg/24 Std.] & 15 & 27,4 & 33,8 & 42,3 & 50,8 & 61,0 \\
Oxalsäure-Clearance [ml/min] & 15 & 8,9 & 15,5 & 18,9 & 25,0 & 37,9 \\
Kreatinin-Clearance [ml/min] & 15 & 93,8 & 104,7 & 116,4 & 123,0 & 144,0 \\
Oxalsäure-Clearance/Kreatinin-Clearance & 15 & 0,09 & 0,12 & 0,15 & 0,23 & 0,33 \\
\hline
\end{tabular}

Tab.' 2: Meßergebnisse bei niereninsuffizienten Patienten

\begin{tabular}{|c|c|c|c|c|c|c|}
\hline & $n$ & Minimum & $1 \mathrm{~s}$ & Median & $3 \mathrm{~S}$ & Maximum \\
\hline $\begin{array}{l}\text { Oxalsäurekonzentration im Plasma vor Dialyse }[\mathrm{mg} / \mathrm{dl}] \\
\text { Oxalsäurekonzentration im Plasma vor Dialyse }[\mathrm{mg} / \mathrm{dl}] \\
\text { Oxalsäurekonzentration im Plasma nach Dialyse [mg/dl] } \\
\text { Abfall der Plasmakonzentration durch Dialyse auf [\%] }\end{array}$ & $\begin{array}{l}25 \\
17 \\
17 \\
17\end{array}$ & $\begin{array}{c}0,39 \\
0,75 \\
0,40 \\
43,9\end{array}$ & $\begin{array}{l}0,75 \\
0,96 \\
0,61 \\
52,7\end{array}$ & $\begin{array}{l}0,98 \\
1,21 \\
0,67 \\
56,3\end{array}$ & $\begin{array}{r}1,28 \\
1,29 \\
0,69 \\
63,4\end{array}$ & $\begin{array}{r}1,36 \\
1,36 \\
0,94 \\
85,1\end{array}$ \\
\hline
\end{tabular}

Tab. 3: Meßergebnisse bei niereninsuffizienten Patienten mit Restharnausscheidung

\begin{tabular}{|c|c|c|c|c|c|c|}
\hline & n & Minimum & $1 \mathrm{~s}$ & Median & 35 & Maximum \\
\hline $\begin{array}{l}\text { Oxalsäureausscheidung im Urin [mg/24 Std.] } \\
\text { Oxalsäure-Clearance }[\mathrm{ml} / \mathrm{min}] \\
\text { Kreatinin-Clearance }[\mathrm{ml} / \mathrm{min}] \\
\text { Oxalsäure-Clearance/Kreatinin/Clearance }\end{array}$ & $\begin{array}{l}10 \\
10 \\
10 \\
10\end{array}$ & $\begin{array}{l}1,1 \\
0,06 \\
0,05 \\
0,42\end{array}$ & $\begin{array}{r}10,4 \\
0,86 \\
0,83 \\
1,10\end{array}$ & $\begin{array}{l}17.3 \\
1,68 \\
0.91 \\
1,42\end{array}$ & $\begin{array}{c}28,5 \\
1,96 \\
1,45 \\
2,33\end{array}$ & $\begin{array}{r}38,1 \\
3,78 \\
3,54 \\
2,61\end{array}$ \\
\hline
\end{tabular}




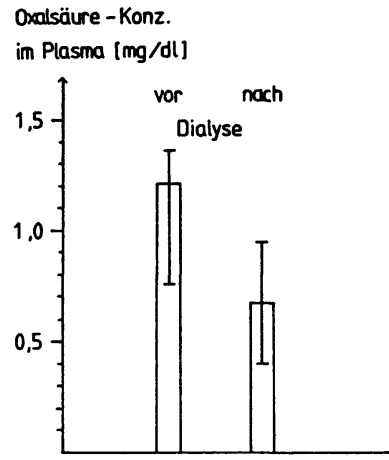

Abb. 4: Oxalsäure-Konzentration im Plasma bei Patienten mit terminaler Niereninsuffizienz ( $n=17$ ) vor und nach Dialyse. Dargestellt sind Medianwert, Minimum und Maximum
Patienten mit terminaler Niereninsuffizienz weisen gegenüber Gesunden signifikante Erhöhungen der Oxalsäurekonzentration im Plasma auf. Neben der Niereninsuffizienz ist hierfür möglicherweise auch eine durch die Urämie gesteigerte Syntheserate aus Präkursoren verantwortlich. Ebenso wie Leber (13) mit einem isotachophoretischen Verfahren zur Bestimmung der Oxalsäurekonzentration finden auch wir postdialytisch eine Erniedrigung der Konzentration auf $56 \%$. Daraus ergibt sich, daß Oxalsäure als kleinmolekulare Substanz dialysierbar ist.

Der bei terminaler Niereninsuffizienz gegenüber der KreatininClearance deutlich geringere Abfall der Oxalsäure-Clearance ist wahrscheinlich darauf zurückzuführen, daß Oxalsäure nicht nur glomerulär filtriert wird, sondern entweder durch passive lonendiffusion (14) oder durch Sekretion im proximalen Tubulus, wie Weinman (15) durch Mikropunktion an Ratten nachweist, ausgeschieden wird. Die höchsten Plasmakonzentrationen der Oxalsäure finden wir bei zwei Patienten mit primärer Hyperoxalurie. Bestimmungen der Oxalsäure im Plasma können somit bei Verdacht auf Oxalose, insbesondere wenn die Oxalatausscheidung in Folge der fortschreitenden Niereninsuffizienz in den Normbereich abfälit, zur Sicherung der Diagnose herangezogen werden.

\section{Oxalose}

Bei zwei Patienten mit nachgewiesenen Oxalatniederschlägen in Geweben betragen die Oxalsäurekonzentrationein im Plasma $2,65 \mathrm{mg} / \mathrm{dl}$ und $1,37 \mathrm{mg} / \mathrm{dl}$. Die letztere Patientin scheidet $323,7 \mathrm{mg}$ Oxalsäure/24 Std. aus (Abb. 3).

\section{Diskussion}

Die von uns gemessenen Oxalsäurekonzentrationen im Plasma stimmen mit den Meßergebnissen überein, die andere Untersucher mit technisch und zeitlich aufwendigeren $\mathrm{Me}$ thoden finden: Bennett ( 8 ) mit einer Isotopenverdünnungsmethode $(0,046-0,141 \mathrm{mg} / \mathrm{dl}$ im Plasma), Hatch (9) enzymatisch mit Formiat-Dehydrogenase $(0,07-0,19 \mathrm{mg} / \mathrm{dl} \mathrm{im}$ Serum), Krugers Dagneaux (10) kolorimetrisch mit Chromotropsäure $(0,117-0,250 \mathrm{mg} / \mathrm{dl}$ im Plasma) sowie Koh/becker und Butz (11) mit Oxalat-Oxidase in einer Katalase-Reaktion $(0,15-0,40 \mathrm{mg} / \mathrm{dl}$ im Serum).

Bei der Bestimmung der Oxalsäurekonzentration im Plasma muß berücksichtigt werden, daß sich sofort nach der Blutentnahme die Konzentration durch Neubildung aus dem Präkursor Glyoxylsäure erhöhen kann (6), was Akcay (7) hạt nachweisen können. Daher können sich zu hohe Konzentrationen ergeben. Die Reaktion Glyoxylsäure $\rightarrow$ Oxalsäure wird durch die Enzyme Xanthin-Oxidase, Glykolat-Oxidase und Laktat-Dehydrogenase katalysiert (6), die wiederum durch Allopurinol, Phenylmilchsäure und Borsäure gehemmt werden können (7). Ohne Inhibitoren dieser Reaktion steigt die Oxalsäurekonzentration in 7 Tagen auf etwa den dreifachen Ausgangswert an (Abb. 2). Anschließend scheint sich die Konzentration nicht mehr entscheidend zu erhöhen, was damit zu erklären ist, daß Oxalsäure selbst wiederum GlykolatOxidase und Laktat-Dehydrogenase hemmt. So ist es bei Zusatz der Inhibitoren nicht mehr erforderlich, die Proben sofort zu untersuchen. Dadurch wird die klinische Praktikabilität erhöht.
Schrifttum:

1. SUGIURA, M. et al.: Enzymic determination of serum oxalate. Clin. Chim. Acta 105, $393-$ 399 (1980).

2. ARCHER, H. E, DORMER, A. E., SCOWEN, E. F., WATTS, R. W. E.: Studies on the urinary excretion of oxalate by normal subjects. Clin. Sci. (London) 16, 405-411 (1957).

3. HAUSMAN, E. R., MC ANALLY, J. S., LEWIS, G. T.: Determination of oxalate in urine.

3lin. Chem. (New York) 2, $439-444$ (1956).

4. HODGKINSON, A., WILLIAMS, A.: An improved color
Clin. Chim. Acta (Amsterdam) 36, 127-132 (1972)

5. GOMORI, G COLOWICK and KAPLAN: Meth. in Enzymol. (New York) Bd. 1, 138

(1955).

6. HODGKINSON, A.: Oxalic Acid in Biology and Medicine, Academic Press London, 126

(1977).

7. AKCAY, T., R

8. BENNETT, D.

J. et al.: A radioenzymatic isotope-dilution assay for oxalate in serum or plasma, Clin. Chem. 25/10, 1810-1813 (1979)

HATCH, M., BOURKE, E., COSTELLO, J.: New enzymatic method for serum oxalate determination. Clin. Chem. 23/1, 76-78 (1977).

10. KRUGERS DAGNEAUX, P. G. L C. et al.: Oxalic acid determination in plasma. Clin. Chim. Acta 71, 319-325 (1976).

11. KOHLBECKER, G., BUTZ M.: Direct spectrophotometric determination of serum and urinary oxalate with oxalate oxidase. J. Clin. Chem. Clin. Biochem., Vol. 19. 1103-1106

12. ZAREMBSKI, P. M., HODGKINSON, A.: The renal clearance of oxalic acid in normal subjects and in patients with primary hyperoxaluria. Investigative Urology 1, 87-93 (1963) 13. LEBER, H. W., MUNZEL, U., RAWER, P. et al.: Oxalsaurestoffwechsel bei chronische Uramie: Untersuchungen uber das Verhalten der Oxalsaure im Plasma von Dialysepatienten. Verhandlungen der Deutschen Gesellschaft für Innere Medizin, 87. Kongreß, 717-722
(1981).

14. HAUTMANN, R. OSSWALD, H.: Pharmacokinetic studies of oxalate in man. Invest Urol. 16, 395-398 (1979)

15. WEINMAN, E.J.FRANKFURT, S. J,INCE, A, SANSOM, S.: Renal tubular transport of organic acids. Studies with oxalate and para-aminohippurate in the rat. J. Clin. Invest. 61, $801-806$ (1978).

Für die Verfasser:

Prof. Dr. W. Ruge

Medizinische Klinik im Krankenhaus Oststadt

der Medizinischen Hochschule Hannover

Podbielskistraße 380

D-3000 Hannover 51 